\title{
Revista Colombiana de

\section{Guía de práctica clínica para la prevención, detección temprana, diagnóstico, tratamiento y seguimiento de las dislipidemias: tratamiento farmacológico con estatinas}

\author{
Oscar Múñoz ${ }^{a}$, Ángel A. García ${ }^{\mathrm{b}, *}$, Daniel G. Fernández ${ }^{\mathrm{b}}$, Angélica M. Higuera ${ }^{\mathrm{b}}$, \\ Álvaro J. Ruiz ${ }^{\mathrm{C}}$, Pablo Aschner ${ }^{\mathrm{d}}$, Juan M. Toro ${ }^{\mathrm{e}}$, Juan M. Arteaga ${ }^{\mathrm{f}}$, \\ Alonso Merchán ${ }^{\mathrm{g}}$, Gregorio Sánchez Vallejo ${ }^{\mathrm{h}}$ e Yadira Villalba ${ }^{\mathrm{i}}$
}

\footnotetext{
a Unidad de Clínica Hospitalaria, Departamento de Epidemiología Clínica y Bioestadística, Hospital Universitario de San Ignacio, Pontificia Universidad Javeriana, Bogotá, Colombia

b Unidad de Cardiología, Departamento de Epidemiología Clínica y Bioestadística, Hospital Universitario de San Ignacio, Pontificia Universidad Javeriana, Bogotá, Colombia

c Departamento de Medicina Interna, Departamento de Epidemiología Clínica y Bioestadística, Pontificia Universidad Javeriana, Bogotá, Colombia

¿ Departamento de Medicina Interna, Pontificia Universidad Javeriana, Hospital Universitario San Ignacio, Bogotá, Colombia

e Departamento de Medicina Interna, Universidad de Antioquia, Hospital Pablo Tobón Uribe, Antioquia, Colombia

${ }^{f}$ Departamento de Medicina Interna, Universidad Nacional de Colombia, Bogotá, Colombia

s Sociedad Colombiana de Cardiología y Cirugía Cardiovascular, Fundación Clínica Shaio, Bogotá, Colombia

h Departamento de Medicina Interna, Hospital Departamental Universitario del Quindio San Juan de Dios, Quindío, Colombia

' Departamento de Medicina Interna, Universidad de Cartagena, Cartagena, Colombia
}

PALABRAS CLAVE

Hipercolesterolemia;

Lípidos;

Tratamiento;

Estatinas

\section{Resumen}

Objetivo: Evaluar el impacto del tratamiento con estatinas sobre la incidencia y la recurrencia de los eventos cardiovasculares y los cerebrovasculares (prevención primaria y secundaria), los niveles de las fracciones lipídicas y la incidencia de efectos secundarios (el cáncer y la diabetes mellitus) en personas con hipercolesterolemia.

\footnotetext{
* Autor para correspondencia.

Correo electrónico: angel.garcia@javeriana.edu.co (Á.A. García).
} 
Métodos: Se elaboró una guía de práctica clínica siguiendo los lineamientos de la guía metodológica del Ministerio de Salud y Protección Social para recolectar de forma sistemática la evidencia científica y formular las recomendaciones utilizando la metodología GRADE.

Resultados: Se evidenció un efecto benéfico del tratamiento farmacológico con estatinas tanto en población de prevención primaria como en prevención secundaria, logrando reducciones clínica y estadísticamente significativas en la mortalidad y en los eventos cardiovasculares y cerebrovasculares. Adicionalmente, el tratamiento intensivo con estatinas mostró mayor reducción en los eventos cardiovasculares y cerebrovasculares ateroscleróticos al compararlo con el tratamiento de intensidad moderada. No se encontraron diferencias significativas en el riesgo de desarrollar cáncer al comparar estatinas frente a placebo pero sí se encontró un incremento de nueve por ciento en el riesgo de presentar diabetes mellitus asociado al tratamiento con estatinas.

Conclusiones: Se formulan recomendaciones a favor del uso de estatinas como primera línea de tratamiento de hipercolesterolemia, y se establecen criterios para definir la intensidad de la terapia (alta o moderada respuesta) según el riesgo cardiovascular a 10 años, el nivel de colesterol LDL (CLDL), la edad y los antecedentes personales y los familiares.

(C) 2014 Sociedad Colombiana de Cardiología y Cirugía Cardiovascular. Publicado por Elsevier España, S.L.U. Este es un artículo Open Access bajo la licencia CC BY-NC-ND (http://creativecommons.org/licenses/by-nc-nd/4.0/).

\section{KEYWORDS}

Hypercholesterole-

mia;

Lipid;

Treatment;

Statins
Clinical practice guidelines for prevention, early detection, diagnose, treatment and follow-up of dyslipidemias: farmacological treatment with statins

\begin{abstract}
Objective: To evaluate the impact of statin therapy on the incidence and recurrence of cardiovascular and cerebrovascular events (primary and secondary prevention), the levels of lipid fractions and the incidence of side effects (cancer and diabetes mellitus) in people with hypercholesterolemia.

Methods: A clinical practice guideline was developed following the guidelines of the methodological guidance of the Ministry of Health and Social Protection to systematically collect the evidence and make recommendations using the GRADE methodology.

Results: A beneficial effect of pharmacological treatment with statins in both primary prevention and secondary prevention was evident, achieving clinically and statistically significant reductions in mortality and cardiovascular and cerebrovascular events. Additionally, intensive statin therapy showed greater reduction in atherosclerotic cardiovascular and cerebrovascular events as compared to the treatment of moderate intensity. No significant differences in the risk of developing cancer by comparing statins versus placebo were found, but a $9 \%$ increase in the risk of diabetes mellitus associated with statin therapy was found.

Conclusions: Recommendations for the use of statins as first-line treatment of hypercholesterolemia are formulated, and criteria to define the intensity of therapy (high or moderate response) as cardiovascular risk at 10 years, level of LDL (LDLc), age and personal and family history were established.

(c) 2014 Sociedad Colombiana de Cardiología y Cirugía Cardiovascular. Published by Elsevier España, S.L.U. This is an open access article under the CC BY-NC-ND license (http://creativecommons.org/licenses/by-nc-nd/4.0/).
\end{abstract}

\section{Introducción}

La enfermedad cardiovascular es una de las principales causas de morbilidad y la primera causa de mortalidad a nivel mundial. Dentro del amplio espectro de esta entidad se encuentran la enfermedad aterosclerótica y la enfermedad cerebrovascular, con el infarto agudo del miocardio y el ataque cerebrovascular como los que generan la mayor carga de mortalidad ${ }^{1}$.

Los estudios observacionales han mostrado una relación directamente proporcional entre el riesgo de enfermedad coronaria y las concentraciones séricas de colesterol $^{2-4}$, identificando a la hipercolesterolemia como un factor de riesgo independiente para los eventos cardiovasculares y los cerebrovasculares en personas con o sin historia de enfermedad cardiovascular ${ }^{5}$. Por lo cual, la corrección del perfil lipídico, especialmente del colesterol de baja densidad (cLDL), constituye un objetivo terapéutico importante ${ }^{1}$.

Las estatinas han sido ampliamente usadas para mejorar la sobrevida y reducir la incidencia de los eventos coronarios y los eventos cerebrovasculares en pacientes con enfermedad cardiovascular, y se constituyen en el grupo 
Tabla 1 Intensidad de la terapia farmacológica con estatinas

\begin{tabular}{ll}
\hline Moderada intensidad & Alta intensidad \\
\hline Reducción de cLDL entre $30-50 \%$ & Reducción de cLDL $\geq 50 \%$ \\
Atorvastatina $10,20,40 \mathrm{mg}$ & Atorvastatina $80 \mathrm{mg}$ \\
Rosuvastatina $5,10,20 \mathrm{mg}$ & Rosuvastatina $40 \mathrm{mg}$ \\
Pravastatina $40 \mathrm{mg}$ & \\
Simvastatina $20,40 \mathrm{mg}$ & \\
Lovastatina $20,40 \mathrm{mg}$ & \\
\hline
\end{tabular}

La intensidad de la terapia con estatinas se define según el porcentaje de reducción de LDL respecto a la concentración basal del individuo.

farmacológico de primera elección para la disminución del $\mathrm{CLDL}^{6}$.

Aunque inicialmente la terapia con estatinas se enfocó en la prevención secundaria, con el paso del tiempo el tratamiento se ha indicado en pacientes con riesgo cardiovascular progresivamente más bajo, incrementando así el número de personas candidatas a recibir manejo farmacológico con estatinas ${ }^{7}$.

Existen múltiples estudios que demuestran el efecto favorable del tratamiento con estatinas en poblaciones de alto riesgo ${ }^{8-19}$, sin embargo, aún se discute su beneficio en la reducción del riesgo cardiovascular en personas sin evidencia de enfermedad cardiovascular ${ }^{20}$.

Anteriormente las poblaciones de bajo riesgo se definían por la ausencia de enfermedad arterial coronaria, pero se ha reconocido que en estos grupos se incluye tanto a personas con alto riesgo de desarrollar enfermedad coronaria (por ejemplo, aquellos con enfermedad vascular periférica severa) como a personas con riesgo muy bajo (por ejemplo, personas jóvenes, sin enfermedades como la diabetes mellitus o la hipertensión arterial y los niveles de colesterol $\mathrm{cLDL}<70 \mathrm{mg} / \mathrm{dl}$ ). Por lo anterior, actualmente el uso de estatinas está basado en el riesgo estimado de presentar un evento aterosclerótico y no únicamente en la presencia o ausencia de enfermedad coronaria conocida ${ }^{21-23}$.

Los resultados del estudio Jupiter ${ }^{24}$, incentivaron el uso de estatinas en personas sin historia de enfermedad coronaria y plantearon la discusión sobre el mayor impacto clínico de las estatinas de alta potencia frente a las de baja potencia. Recientemente, el uso de estatinas en esta población también ha sido evaluado por la colaboración Cholesterol Treatment Trialistsćollaborators, mostrando una reducción de $21 \%$ en el riesgo relativo de eventos vasculares mayores con el uso de estatinas, por cada $1,0 \mathrm{mmol} / \mathrm{L}(38 \mathrm{mg} / \mathrm{dl})$ de disminución en el cLDL, independientemente del riesgo basal $^{25}$, confirmando la eficacia de las estatinas en prevención primaria.

Otros estudios diseñados para determinar la seguridad de las reducciones mayores del colesterol LDL y el impacto sobre los eventos vasculares, comparando esquemas de tratamiento con diferente intensidad, han sugerido mayor beneficio al emplear terapia más intensiva ${ }^{26-30}$.

En la tabla 1 se presenta la clasificación de la terapia con estatinas según su intensidad.

Teniendo en cuenta estos resultados, es relevante evaluar el impacto clínico del tratamiento con estatinas tanto en la población de prevención primaria como secundaria, así como la intensidad del mismo, para determinar el momento oportuno de inicio de la terapia farmacológica y el esquema que se deba emplear.

El Ministerio de Salud y Protección Social ha encargado a la Pontificia Universidad Javeriana y a la Alianza CINETS conformada por las Universidades Javeriana, Nacional y Antioquia el desarrollo de una guía clínica sobre la prevención, detección temprana, diagnóstico, tratamiento y seguimiento de las dislipidemias en la población mayor de 18 años. El resultado de ese proceso, en lo referente al manejo farmacológico con estatinas en adultos con dislipidemia se presenta de forma resumida en el presente escrito. Cabe resaltar que en este proceso también participaron activamente la Asociación Colombiana de Medicina Interna, la Sociedad Colombiana de Cardiología, y la Asociación Colombiana de Endocrinología.

\section{Métodos}

El proceso de desarrollo de la guía se describe detalladamente en el manual para desarrollo de guías de práctica clínica y en la actualización de dicha metodología (disponible en la página web del Ministerio de Salud y Protección Social) ${ }^{31}$.

Cabe resaltar que dicha metodología favorece la búsqueda sistemática de la evidencia científica (incluyendo tanto las revisiones sistemáticas de la literatura como los estudios primarios). Así mismo, establece una metodología clara para la selección de la evidencia que se debe utilizar, evaluando cuidadosamente la calidad de la misma. La versión completa de la guía, en la que se presentan los resultados de todas estas evaluaciones, pueden ser consultados en la página web del Ministerio de Salud y Protección Social, así como en la página web de la alianza CINETS.

El grupo metodológico preparó un resumen de la evidencia disponible y lo presentó al panel completo durante las reuniones de generación de recomendaciones. Este panel incluyó a representantes de las diversas especialidades médicas (cardiólogos, endocrinólogos, médicos internistas, médicos de familia, médicos generales) así como a otros profesionales de la salud (nutricionistas, terapeutas físicos). Cabe resaltar que durante todo el proceso se contó adicionalmente con representantes de los pacientes, que aportaron activamente la generación de recomendaciones. Todos los integrantes del panel presentaron abiertamente la declaración de sus conflictos de intereses. Estos documentos están disponibles en la versión completa de la guía.

Durante las reuniones de generación de recomendaciones se siguió la metodología propuesta por el grupo GRADE, que permite tener en cuenta no solo la calidad de la evidencia disponible, sino consideraciones de los costos, las preferencias de los pacientes y la relación entre los beneficios y los riesgos de las tecnologías de interés (las pruebas, las estrategias de manejo, las intervenciones, los medicamentos). Las recomendaciones presentadas muestran por tanto la fuerza de la recomendación (la fuerte o la débil), la dirección de la misma (a favor o en contra de la intervención) así como la calidad de la evidencia que la soporta (muy baja, baja, moderada, alta o consenso de expertos).

La versión final de la guía fue evaluada por pares internacionales, seleccionados por el Ministerio de la Protección 
Social, expertos tanto en dislipidemias como en metodología. Sus aportes fueron tenidos en cuenta por el grupo desarrollador de la guía.

\section{Resultados}

Se condujo una búsqueda sistemática de la literatura de novo para identificar metaanálisis o revisiones sistemáticas de la literatura publicadas entre enero de 2008 y julio de 2013, empleando las bases de datos sugeridas en la guía metodológica (MEDLINE, Cochrane, CRD Database). Se identificaron 25 metaanálisis que abordan el impacto del tratamiento con estatinas sobre los desenlaces seleccionados (la mortalidad, la angina, el infarto agudo del miocardio, el ataque cerebrovascular, los niveles de las fracciones lipídicas, el cáncer, la diabetes mellitus) ${ }^{1,20,25,32-53}$. Adicionalmente, por sugerencia de los expertos emáticos se incluyó otro metaanálisis ${ }^{54}$, que no fue identificado en las búsquedas realizadas.

Para la población de prevención primaria, al comparar el uso de estatinas frente a placebo se encontró una reducción de la mortalidad global de $14 \%$ a favor del grupo en tratamiento con estatinas (OR 0,86, IC 95\% 0,79;0,94) ${ }^{1}$, así como una disminución de $22 \%$ en el riesgo de presentar ataque cerebrovascular con el uso de estatinas (RR 0,78, IC 95\% $0,68 ; 0,89)^{1}$. También se halló una reducción de $37 \%$ en el riesgo de presentar infarto agudo del miocardio (RR 0,63, IC $95 \% 0,50 ; 0,79)^{20}$ y una reducción de $29 \%$ en el riesgo de presentar angina inestable en los pacientes tratados con estatinas (RR 0,71 IC 95\% 0,55; 0,92) ${ }^{20}$. Para el desenlace de niveles de las fracciones lipídicas se demostró una reducción de 40,53 mg/dl en el colesterol total (DM -40,53 IC 95\% $-52,11 ;-29,33)$ y de $38,60 \mathrm{mg} / \mathrm{dl}$ en el CLDL (DM $-38,60$, IC $95 \%-44,77 ;-32,81)^{1}$. Todas las diferencias resultan clínica y estadísticamente significativas.

Al evaluar el tratamiento con estatinas frente a placebo en la población de prevención secundaria se encontró una asociación estadística y clínicamente significativa entre el uso de estatinas y reducción de mortalidad, con una disminución de $18 \%$ en la probabilidad de presentar este desenlace (OR 0,82 IC 95\% 0,75; 0,90) $)^{34}$. También se observó una disminución de $16 \%$ en los eventos de ataque cerebrovascular (RR 0,84 IC $95 \% 0,76 ; 0,93$ ) y una reducción de $27 \%$ en el riesgo de presentar infarto agudo del miocardio en los pacientes que recibieron tratamiento con estatinas (RR 0,73 IC 95\% $0,65 ; 0,81)^{35}$; en ambos casos los resultados son clínica y estadísticamente significativos.

El estudio de la colaboración Cholesterol Treatment TrialistsĆollaborators ${ }^{25}$, evaluó la eficacia y la seguridad de la disminución intensiva del cLDL por medio del tratamiento con estatinas. Al comparar terapia intensiva frente a menos intensiva se encontró que con una reducción de $38,6 \mathrm{mg} / \mathrm{dl}$ en los niveles de cLDL se logra una disminución del $29 \%$ en los eventos de infarto agudo del miocardio no fatal (RR 0,71 IC 95\% 0,58; 0,87) y del $26 \%$ en los eventos de ataque cerebrovascular (RR 0,74 IC 95\% 0,59; 0,92).

También se observó que estas reducciones en los eventos vasculares no dependen de la concentración basal de cLDL, y se logran reducciones significativas en el riesgo de presentar estos eventos en todos los casos en los que se disminuyó el cLDL con terapia intensiva. Para aquellos con reducción de los niveles de cLDL respecto al valor basal $<38,6 \mathrm{mg} / \mathrm{dl}$ se observó una disminución de $29 \%$ en los eventos vasculares mayores (RR 0,71 IC 95\% 0,52; 0,98); en quienes alcanzaron una reducción del cLDL entre 77,2 y $96,5 \mathrm{mg} / \mathrm{dl}$ se observó una disminución de $23 \%$ en dichos eventos (RR 0,77; IC 95\% 0,64; 0,94) y en quienes alcanzaron una reducción del $\mathrm{CLDL} \geq 135,1 \mathrm{mg} / \mathrm{dl}$ los eventos disminuyeron $36 \%$ (RR 0,64 IC $95 \%$ 0,47; 0,86).

No se encontraron diferencias estadísticamente significativas en la mortalidad de origen coronario al comparar la terapia intensiva con estatinas frente a la terapia menos intensiva (RR 0,85 IC 95\% 0,63; 1,15).

En cuanto a los efectos adversos, al comparar frente a placebo, no se encontró asociación entre el uso de estatinas y riesgo aumentado de desarrollar cáncer (OR 0,96, IC 95\% $0,91 ; 1,02)^{54}$. Sí se encontraron diferencias de estadística y clínicamente significativas en la ocurrencia de diabetes mellitus, mostrando $9 \%$ más chance de presentar este desenlace (OR 1,09, IC 95\% 1,02; 1,16) en aquellos pacientes que estaban recibiendo estatinas.

\section{Discusión}

De acuerdo con la información presentada, resulta evidente el efecto benéfico del tratamiento farmacológico con estatinas tanto en la población de prevención primaria como en prevención secundaria, logrando reducciones clínica y estadísticamente significativas en la mortalidad y en los eventos cardiovasculares y los cerebrovasculares (el infarto agudo del miocardio, el ataque cerebrovascular y la angina).

También se encontró fuerte evidencia, aportada por la colaboración Cholesterol Treatment TrialistsĆollaborators ${ }^{25}$, a favor del tratamiento intensivo con estatinas, mostrando mayor reducción en los eventos ateroscleróticos al compararlo con el tratamiento de intensidad moderada. Adicionalmente, se observó que la reducción en el riesgo relativo de los eventos cardiovasculares o los cerebrovasculares es independiente de la concentración basal de cLDL y que la reducción absoluta en el cLDL se asocia con una reducción proporcional en el riesgo cardiovascular. De tal forma que al definir la intervención a realizar en el ámbito clínico, se debería buscar una reducción del CLDL basado en el riesgo cardiovascular calculado a 10 años para cada paciente y no en la consecución de metas sobre las cuales no hay evidencia de impacto en prevención de los eventos cardiovasculares o los cerebrovasculares.

Esta intervención permitiría diferenciar el perfil de riesgo de los pacientes y según esto se definiría la intensidad apropiada del tratamiento para lograr una reducción significativa en el riesgo de presentar eventos cardiovasculares ateroscleróticos. Así mismo, en quienes tengan un riesgo cardiovascular bajo (< 10\% según la escala de Framingham ajustada para la población colombiana), habría que considerar la relación entre riesgos y beneficios para definir el inicio del tratamiento con estatinas en dosis moderada o realizar modificaciones en el estilo de vida.

Respecto a los eventos adversos que fueron evaluados, no se encontraron diferencias significativas en el riesgo de desarrollar cáncer al comparar estatinas frente a placebo pero se encontró un incremento de $9 \%$ en el riesgo de presentar diabetes mellitus asociado con el tratamiento con 
estatinas. Sin embargo, se encontraron serias limitaciones metodológicas que comprometen la calidad de la evidencia de este último desenlace ya que la incidencia de la diabetes no ha sido un desenlace primario en la mayoría de los grandes estudios realizados sobre el uso de estatinas y no en todos se realizaron mediciones basales de glucemia. Por otra parte, no se especifica un método consistente para establecer el diagnóstico, por lo que pacientes con o sin evidencia bioquímica de diabetes podrían ser categorizados como nuevos casos de diabetes y realmente corresponder a casos no diagnosticados previamente. Por tanto, aunque esta ha sido una alternativa para evaluar la incidencia de diabetes mellitus asociada al uso de estatinas, se requiere mayor información para analizar la relación causa-efecto. $Y$ aun si el aumento es real, es de una magnitud claramente inferior al beneficio potencial del tratamiento con estatinas.

Vale la pena tener en cuenta que durante el proceso de elaboración de la guía se publicó la versión preliminar de la guía para el tratamiento del colesterol para reducir el riesgo cardiovascular aterosclerótico en adultos del Colegio Americano de Cardiología y de la Asociación Americana del Corazón (ACC/AHA 2013) ${ }^{55}$, se evaluó su calidad y se consideró apta para ser incluida como fuente de evidencia.

Esta guía se centra en emplear la intensidad apropiada en la terapia farmacológica con estatinas para reducir el riesgo cardiovascular en quienes más probablemente se beneficien de dicha intervención. Los autores de esta guía recalcan que las fuentes de evidencia disponibles incluyeron experimentos clínicos controlados que compararon dosis fijas de estatinas frente a placebo o ningún tratamiento, y aquellos que compararon dosis fijas de estatinas de intensidad alta frente a estatinas de intensidad moderada. Sin embargo, no se encontraron estudios diseñados para evaluar el impacto del tratamiento con dosis ajustadas de estatinas para lograr metas específicas de cLDL o colesterol no HDL, por lo cual no hay evidencia que soporte esta intervención que previamente se había recomendado en las guías del ATP $1 I^{6}$.

Las guías ACC/AHA $2013^{55}$, recomiendan el inicio de terapia con estatinas en personas con riesgo cardiovascular incrementado, pues son quienes tienen mayor probabilidad de beneficiarse en términos de la reducción del riesgo cardiovascular, aun al considerar los posibles efectos adversos asociados con esta intervención. En el caso de prevención primaria de enfermedad cardiovascular aterosclerótica en individuos sin manifestaciones clínicas, el inicio de la terapia con estatinas se determinará de acuerdo con el riesgo cardiovascular estimado a 10 años y en prevención secundaria, la evidencia soporta el tratamiento de alta intensidad con estatinas para disminuir al máximo los niveles de cLDL.

Estas recomendaciones son consistentes con los resultados hallados tras el proceso de búsqueda y recolección sistemática de la evidencia y con las recomendaciones formuladas por el panel de expertos que participaron en la elaboración de la guía de práctica clínica para la prevención, detección temprana, diagnóstico, tratamiento y seguimiento de las dislipidemias.

\section{Conclusiones}

A continuación se presentan las recomendaciones finales de la guía de práctica clínica para la prevención, detección temprana, diagnóstico, tratamiento y seguimiento de las dislipidemias, en cuanto al tratamiento farmacológico con estatinas. Dichas recomendaciones fueron derivadas de la evidencia presentada y las consideraciones mostradas en la discusión. La información completa del proceso para generar las recomendaciones se encuentra en la versión completa de la guía disponible a través de la página electrónica del Ministerio de Salud y Protección Social y en la página electrónica de la Alianza CINETS.

Esta misma información se presenta a manera de algoritmo en la figura 1.

\section{Recomendaciones}

1. Se recomienda el inicio de terapia con estatinas de alta intensidad ${ }^{\mathrm{a}}$ en:

- Personas con historia de enfermedad cardiovascular ateroscleróticab.

- Personas con nivel de cLDL > $190 \mathrm{mg} / \mathrm{dl}$.

- Personas con diabetes mellitus, mayores de 40 años, que tengan un factor de riesgo cardiovascular asociado $^{c}$ y cLDL > $70 \mathrm{mg} / \mathrm{dl}$.

- Personas con estimación de riesgo cardiovascular $>10 \%$ a 10 años, según la escala de Framingham recalibrada para Colombia.

Recomendación fuerte a favor de la intervención. Calidad de la evidencia: $\oplus \oplus \oplus \oplus \oplus$ Alta.

2. Se recomienda el inicio de terapia con estatinas de moderada intensidad ${ }^{\mathrm{C}}$ en:

- Personas con diabetes mellitus, mayores de 40 años, con CLDL > $70 \mathrm{mg} / \mathrm{dl}$ y sin criterios de terapia intensiva. Recomendación fuerte a favor de la intervención. Calidad de la evidencia: $\oplus \oplus \oplus \ominus$ Moderada.

3. Se sugiere considerar terapia con estatinas de moderada intensidad en personas que no quedaron incluidas en ninguno de los grupos anteriores y tengan una o más de las siguientes condiciones:

- Nivel de cLDL > $160 \mathrm{mg} / \mathrm{dl}$.

- Historia familiar de eventos cardiovasculares o cerebrovasculares ateroscleróticos tempranos en familiares de primer grado de consanguinidad (hombres menores de 55 años o mujeres menores de 65 años).

Recomendación débil a favor de la intervención. Calidad de la evidencia: $\oplus \oplus \oplus \ominus$ Moderada.

Punto de buena práctica clínica

- Se deben implementar las estrategias de prevención primaria de diabetes mellitus definidas para la población

\footnotetext{
a En personas con edad mayor de 75 años podrá definirse el uso de estatinas de alta o moderada intensidad de acuerdo con el balance de riesgo-beneficio o la preferencia del paciente.

b Se consideran personas con historia de enfermedad cardiovascular aterosclerótica a aquellas que hayan presentado eventos coronarios agudos (incluyendo IAM y angina estable o inestable), ACV, AIT, historia previa de revascularización (coronaria o a otro nivel) o enfermedad vascular aterosclerótica de miembros inferiores.

c Se consideran factores de riesgo cardiovascular: HTA, obesidad, tabaquismo.
} 


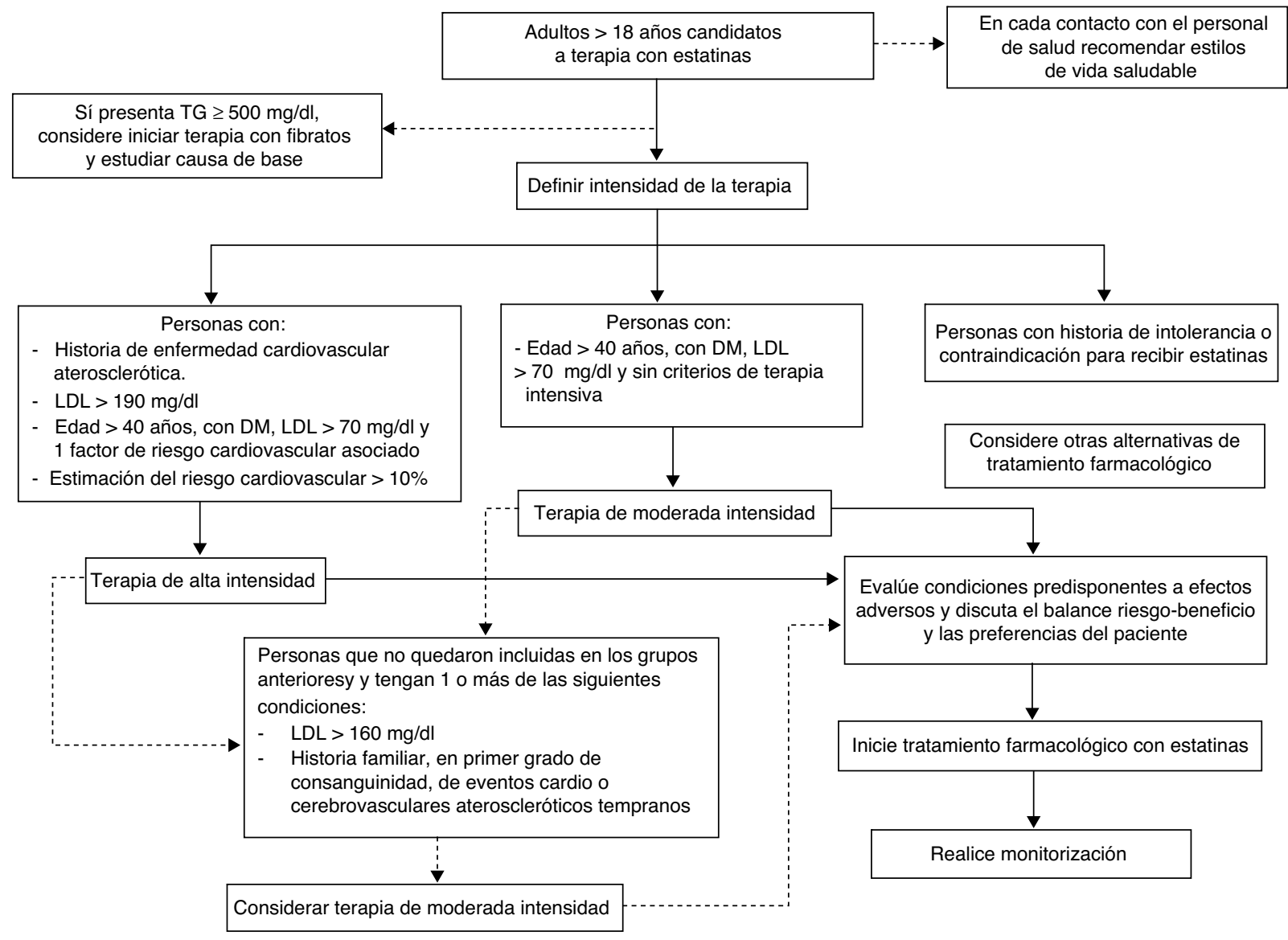

Figura 1 Tratamiento de dislipidemias.

general, como parte de la vigilancia clínica de los pacientes que reciben terapia con estatinas.

\section{Responsabilidades éticas}

Protección de personas y animales. Los autores declaran que para esta investigación no se han realizado experimentos en seres humanos ni en animales.

Confidencialidad de los datos. Los autores declaran que en este artículo no aparecen datos de pacientes.

Derecho a la privacidad y consentimiento informado. Los autores declaran que en este artículo no aparecen datos de pacientes.

\section{Financiación}

El Ministerio de Salud y Protección Social ha encargado a la Pontificia Universidad Javeriana y a la Alianza CINETS conformada por las Universidades Javeriana, Nacional y Antioquia el desarrollo de una Guía clínica sobre la prevención, detección temprana, diagnóstico, tratamiento y seguimiento de las dislipidemias en la población mayor de 18 años.

\section{Conflicto de intereses}

Los autores declaran no tener ningún conflicto de intereses.

\section{Agradecimientos}

Agradecemos la contribución de las personas que, en carácter de representantes de expertos temáticos, usuarios, población blanco o grupos de interés, asistieron o participaron en las reuniones de socialización realizadas durante el desarrollo de la presente guía.

Agradecemos la participación de las instituciones, asociaciones y sociedades científicas, a través de sus representantes, por sus aportes y contribución en los diferentes procesos del desarrollo de la guía:

- Asociación Colombiana de Medicina Interna (ACMI).

- Sociedad Colombiana de Cardiología y Cirugía Cardiovascular.

- Asociación Colombiana de Endocrinología, Diabetes y Metabolismo.

- Asociación Colombiana de Fisioterapia (ASCOFI).

- Centro Colombiano de Nutrición Integral (CECNI).

- Centro Nacional de Investigación en Evidencia y Tecnologías en Salud (Alianza CINETS). 


\section{Bibliografía}

1. Taylor F, Huffman MD, Macedo AF, Moore THM, Burke M, Davey Smith G, et al. Statins for the primary prevention of cardiovascular disease. Cochrane database Syst Rev. 2013;1: CD004816.

2. Stamler J, Vaccaro O, Neaton JD, Wentworth D. Diabetes, other risk factors, and 12-yr cardiovascular mortality for men screened in the Multiple Risk Factor Intervention Trial. Diabetes Care. 1993;16(2):434-44.

3. Chen Z, Peto R, Collins R, MacMahon S, Lu J, Li W. Serum cholesterol concentration and coronary heart disease in population with low cholesterol concentrations. BMJ. 1991;303(6797):276-82.

4. Lewington S, Whitlock G, Clarke R, Sherliker P, Emberson J, Halsey J, et al. Blood cholesterol and vascular mortality by age, sex, and blood pressure: a meta-analysis of individual data from 61 prospective studies with 55,000 vascular deaths. Lancet. 2007;370(9602):1829-39.

5. Prospective Studies Collaboration. Collaborative meta-analysis of 61 studies of vascular risk factors (blood cholesterol, blood pressure, body mass index, diabetes) and cause-specific mortality. Lancet. 2007;370:1829-39.

6. Third Report of the National Cholesterol Education Program (NCEP) Expert Panel on Detection. Evaluation, and Treatment of High Blood Cholesterol in Adults (Adult Treatment Panel III) final report. Circulation. 2002;106(25):3143-421.

7. Last AR, Ference JD, Falleroni J. Pharmacologic treatment of hyperlipidemia. Am Fam Physician. 2011;84(5):551-8.

8. Baigent C, Keech A, Kearney PM, Blackwell L, Buck G, Pollicino C, et al. Efficacy and safety of cholesterol-lowering treatment: prospective meta-analysis of data from 90,056 participants in 14 randomised trials of statins. Lancet. 2005;366:1267-78.

9. Blauw GJ, Lagaay AM, Smelt AH, Westendorp RG. Stroke, statins, and cholesterol. A meta-analysis of randomized, placebo-controlled, double-blind trials with HMG-CoA reductase inhibitors. Stroke. 1997;28:946-50.

10. Briel M, Studer M, Glass TR, Bucher HC. Effects of statins on stroke prevention in patients with and without coronary heart disease: a meta-analysis of randomized controlled trials. Am J Med. 2004;117:596-606.

11. Cheung BMY, Lauder IJ, Lau C-P, Kumana CR. Meta-analysis of large randomized controlled trials to evaluate the impact of statins on cardiovascular outcomes. $\mathrm{Br} \mathrm{J}$ Clin Pharmacol. 2004;57:640-51.

12. Katerndahl DA, Lawler WR. Variability in meta-analytic results concerning the value of cholesterol reduction in coronary heart disease: a meta-meta-analysis. Am J Epidemiol. 1999;149:429-41.

13. LaRosa JC, Applegate W, Crouse JR, Hunninghake DB, Grimm $\mathrm{R}$, Knopp R, et al. Cholesterol lowering in the elderly. Results of the Cholesterol Reduction in Seniors Program (CRISP) pilot study. Arch Intern Med. 1994;154:529-39.

14. Law MR, Wald NJ, Rudnicka AR. Quantifying effect of statins on low density lipoprotein cholesterol, ischaemic heart disease, and stroke: systematic review and meta-analysis. BMJ. 2003;326:1423.

15. Pignone M, Phillips C, Mulrow C. Use of lipid lowering drugs for primary prevention of coronary heart disease: meta-analysis of randomised trials. BMJ. 2000;321:983-6.

16. Thavendiranathan P, Bagai A, Brookhart MA, Choudhry NK. Primary prevention of cardiovascular diseases with statin therapy: a meta-analysis of randomized controlled trials. Arch Intern Med. 2006;166:2307-13.

17. Ward S, Lloyd Jones M, Pandor A, Holmes M, Ara R, Ryan A, et al. A systematic review and economic evaluation of statins for the prevention of coronary events. Health Technol Assess. 2007;11:1-160.
18. Wilt TJ, Bloomfield HE, MacDonald R, Nelson D, Rutks I, Ho M, et al. Effectiveness of statin therapy in adults with coronary heart disease. Arch Intern Med. 2004;164:1427-36.

19. LaRosa JC, He J, Vupputuri S. Effect of statins on risk of coronary disease: a meta-analysis of randomized controlled trials. JAMA. 1999;282:2340-6.

20. Tonelli M, Lloyd A, Clement F, Conly J, Husereau D, Hemmelgarn B, et al. Efficacy of statins for primary prevention in people at low cardiovascular risk: a meta-analysis. CMAJ. 2011;183:E1189-202.

21. D’Agostino RB Sr, Vasan RS, Pencina MJ, Wolf PA, Cobain M, Massaro JM, et al. General cardiovascular risk profile for usein primary care: the Framingham Heart Study. Circulation. 2008;117:743-53.

22. Conroy RM, Pyörälä K, Fitzgerald AP, Sans S, Menotti A, DeBacker G, et al. Estimation of ten year risk of fatal cardiovascular disease in Europe: the SCORE project. Eur Heart J. 2003;24:987-1003, 8.

23. Assmann G, Cullen P, Schulte H. Simple scoring scheme for cal-culating the risk of acute coronary events based on the 10-yearfollow-up of the Prospective Cardiovascular Munster (PROCAM)study. Circulation. 2002;105:310-5, 10.

24. Ridker PM, Danielson E, Fonseca FAH, Genest J, Gotto AM, Kastelein JJ, et al. Rosuvastatin to prevent vascular events in men and women with elevated C-reactive protein. N Engl J Med. 2008;359:2195-207.

25. Baigent C, Blackwell L, Emberson J, Holland LE, Reith C, Bhala $\mathrm{N}$, et al. Efficacy and safety of more intensive lowering of LDL cholesterol: a meta-analysis of data from 170,000 participants in 26 randomised trials. Lancet. 2010;376:1670-81.

26. de Lemos JA, Blazing MA, Wiviott SD. Early intensive vs a delayed conservative simvastatin strategy in patients with acute coronary syndromes: phase $\mathrm{Z}$ of the $\mathrm{A}$ to $\mathrm{Z}$ trial. JAMA. 2004;292:1307-16.

27. Cannon $\mathrm{CP}$, Braunwald $\mathrm{E}, \mathrm{McC}$ abe $\mathrm{CH}$. Intensive versus moderate lipid lowering with statins after acute coronary syndromes. $\mathrm{N}$ Engl J Med. 2004;350:1495-504.

28. Pedersen TR, Faergeman O, Kastelein JJ. High-dose atorvastatin vs usual-dose simvastatin for secondary prevention after myocardial infarction: the IDEAL study: a randomized controlled trial. JAMA. 2005;294:2437-45.

29. LaRosa JC, Grundy SM, Waters DD. Intensive lipid lowering with atorvastatin in patients with stable coronary disease. N Engl J Med. 2005;352:1425-35.

30. Study of the Effectiveness of Additional Reductions in Cholesterol and Homocysteine (SEARCH) Collaborative Group. Intensive lowering of LDL cholesterol with $80 \mathrm{mg}$ versus $20 \mathrm{mg}$ simvastatin daily in 12064 survivors of myocardial infarction: a double-blind randomised trial. Lancet. 2010;376(9753):1658-69.

31. Carrasquilla G, Pulido A, Mieth K, Muñoz O, de la Hoz AM, Guerrero R. Guía metodológica para la elaboración de guías de práctica clínica con evaluación económica en el Sistema General de Seguridad Social en Salud Colombiano. 2013.

32. Adams SP, Tsang M, Wright JM. Lipid lowering efficacy of atorvastatin. Cochrane Database Syst Rev. 2012. CD260082.

33. Weng T-C, Yang Y-HK, Lin S-J, Tai SH. A systematic review and meta-analysis on the therapeutic equivalence of statins. J Clin Pharm Ther. 2010;35:139-51.

34. Naci H, Brugts JJ, Fleurence R, et al. Comparative benefits of statins in the primary and secondary prevention of major coronary events and all-cause mortality: a network meta-analysis of placebo-controlled and active-comparator trials. Eur J Prev Cardiol. 2013;20:641-57.

35. Manktelow BN, Potter JF. Interventions in the management of serum lipids for preventing stroke recurrence. Cochrane Database Syst Rev. 2009. CD 910020.

36. Wlodarczyk J, Sullivan D, Smith M. Comparison of benefits and risks of rosuvastatin versus atorvastatin from a meta-analysis 
of head-to-head randomized controlled trials. Am J Cardiol. 2008;102:1654-62.

37. Mills EJ, O’Regan C, Eyawo O, Wu P, Mills F, Berwanger O, et al. Intensive statin therapy compared with moderate dosing for prevention of cardiovascular events: a meta-analysis of $>40$ 000 patients. Eur Heart J. 2011;32:1409-15.

38. Gutierrez J, Ramirez G, Rundek T, Sacco RL. Statin therapy in the prevention of recurrent cardiovascular events: a sex-based meta-analysis. Arch Intern Med. 2012;172:909-19.

39. Amarenco P, Labreuche J. Lipid management in the prevention of stroke: review and updated meta-analysis of statins for stroke prevention. Lancet Neurol. 2009;8:453-63.

40. Ray KK, Seshasai SRK, Erqou S, Sever P, Jukema JW, Ford I, et al. Statins and all-cause mortality in high-risk primary prevention: a meta-analysis of 11 randomized controlled trials involving 65,229 participants. Arch Intern Med. 2010;170: 1024-31.

41. Bukkapatnam RN, Gabler NB, Lewis WR. Statins for primary prevention of cardiovascular mortality in women: a systematic review and meta-analysis. Prev Cardiol. 2010;13: 84-1390.

42. Alberton M, Wu P, Druyts E, Briel M, Mills EJ. Adverse events associated with individual statin treatments for cardiovascular disease: an indirect comparison meta-analysis. QJM. 2012;105:145-57.

43. Brugts JJ, Yetgin T, Hoeks SE, Gotto AM, Shepherd J, Westendorp RG, et al. The benefits of statins in people without established cardiovascular disease but with cardiovascular risk factors: meta-analysis of randomised controlled trials. BMJ. 2009, 338:b2376.

44. Mills EJ, Wu P, Chong G, Ghement I, Singh S, Akl EA, et al. Efficacy and safety of statin treatment for cardiovascular disease: a network meta-analysis of 170,255 patients from 76 randomized trials. QJM. 2011;104:109-24.

45. Chan DKY, O'Rourke F, Shen Q, Mak JC, Hung WT. Meta-analysis of the cardiovascular benefits of intensive lipid lowering with statins. Acta Neurol Scand. 2011;124:188-95.

46. Afilalo J, Duque G, Steele R, Jukema JW, de Craen AJ, Eisenberg MJ. Statins for secondary prevention in elderly patients: a hierarchical bayesian meta-analysis. J Am Coll Cardiol. 2008;51:37-45.
47. Mills EJ, Rachlis B, Wu P, Devereaux PJ, Arora P, Perri D. Primary prevention of cardiovascular mortality and events with statin treatments: a network meta-analysis involving more than 65,000 patients. J Am Coll Cardiol. 2008;52:1769-81.

48. Jafri H, Alsheikh-Ali AA, Karas RH. Baseline and on-treatment high-density lipoprotein cholesterol and the risk of cancer in randomized controlled trials of lipid-altering therapy. J Am Coll Cardiol. 2010;55:2846-54.

49. Alsheikh-Ali AA, Trikalinos TA, Kent DM, Karas RH. Statins, lowdensity lipoprotein cholesterol, and risk of cancer. J Am Coll Cardiol. 2008;52:1141-7.

50. Mora S, Glynn RJ, Hsia J, MacFadyen JG, Genest J, Ridker PM. Statins for the primary prevention of cardiovascular events in women with elevated high-sensitivity C-reactive protein or dyslipidemia: results from the justification for the use of statins in prevention: an intervention trial evaluating rosuvastatin. Circulation. 2010;121:1069-77.

51. Fernández de Bobadilla J, Moreno R, Fernández C, Martínez A, Sánchez-Maestre C, Ezpeleta-Echevarri D. Effect of intensive treatment with atorvastatin versus standard doses of statins on the risk of stroke. A meta-analysis from five randomized trials including 25,709 patients. Rev Neurol. 2009;48:561-5.

52. Boekholdt SM, Arsenault BJ, Mora S, Pedersen TR, LaRosa JC, Nestel PJ, et al. Association of LDL cholesterol, non-HDL cholesterol, and apolipoprotein B levels with risk of cardiovascular events among patients treated with statins: a meta-analysis. JAMA. 2012;307:1302-9.

53. Sheng X, Wei L, Murphy MJ, MacDonald TM. Statins and total (not LDL) cholesterol concentration and outcome of myocardial infarction: results from a meta-analysis and an observational study. Eur J Clin Pharmacol. 2009;65:1071-80.

54. Naci H, Brugts J, Ades T. Comparative tolerability and harms of individual statins: a study-level network meta-analysis of 246 955 participants from 135 randomized, controlled trials. Circ Cardiovasc Qual Outcomes. 2013;6:390-9.

55. Stone NJ, Robinson J, Lichtenstein AH, Bairey Merz CN, Blum $\mathrm{CB}$, Eckel RH, et al. ACC/AHA Guideline on the treatment of blood cholesterol to reduce atherosclerotic cardiovascular risk in adults: a report of the American College of Cardiology/American Heart Association Task Force on practice guidelines. J Am Coll Cardiol. 2014;63 25 Pt B:2889-934. 